\title{
Improving understanding of RNA structures with the PHENIX/AMBER interface
}

\author{
Jonathon Gray and David Case ${ }^{\mathrm{b}}$ \\ ${ }^{a}$ Rutgers University, Dept. of Chem. and Chem. Biology, Piscataway, NJ 08854, jgg75@chem.rutgers.edu \\ ${ }^{b}$ Rutgers University, Dept. of Chem. and Chem. Biology, Piscataway, NJ 08854, david.case@rutgers.edu
}

In the last decade and a half, many RNA structures have been solved, illuminating some of the functions of different types of RNAs [1-3]. As scientists probe the use of microRNAs as anti-tumor agents and ribozymes, catalytic RNAs, as the basis of the "RNA world" hypothesis, geometrically and physically accurate structures become increasingly important. However, many geometric issues crop up in the structures deposited in the PDB [1,4-5].

While methods like ERRASER have been developed to improve such geometric inaccuracies [1], the PHENIX/AMBER interface, which uses a force field to provide geometric restraints, provides advantages over conventional geometric restraints and ERRASER. These include the ability to perform reciprocal space refinement (in contrast to ERRASER's real space refinement) and the use of physical molecular mechanics force fields that include electrostatic energy terms, which are of great importance with charged molecules like RNA.

To test the effectiveness of this interface on nucleic acids, a set of 19 RNA structures ranging in resolution from 1.32 to $3.6 \AA$, curated from the ERRASER set [1], were prepared for use with the AMBER force fields, and then refined using conventional restraints and AMBER force fields in separate refinements. Upon analysis, the structures refined in conjunction with the AMBER force fields provide more physically-accurate structures with fewer clashes and better energetics, especially at low resolution. Further work performing crystal simulations of these structures has also been performed to gain a better understanding of the structures.

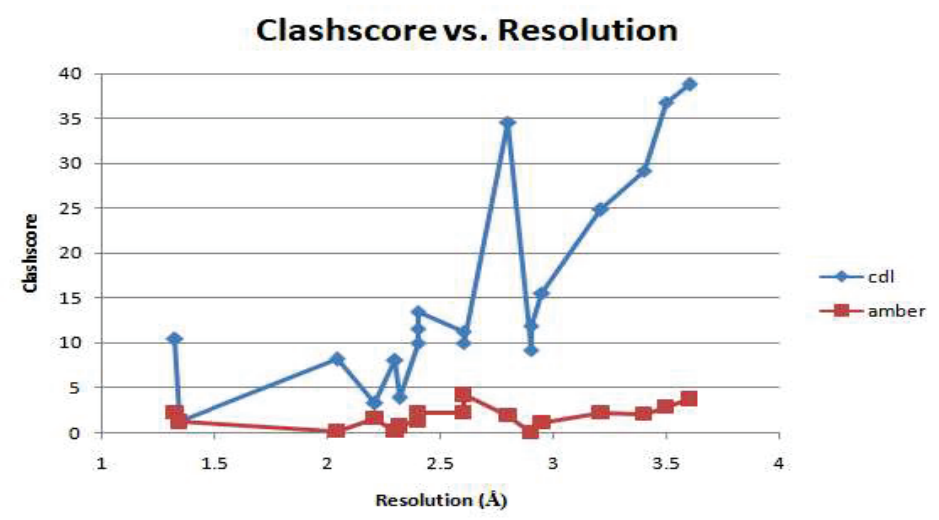

Fig. 1. Comparison of clashscores for refinement of structures using conventional restraints (cdl) and AMBER force fields (amber).

\section{References}

[1] Chou, Fang-Chieh, et al. (2013). Nature Methods, 10, 74-76.

[2] Ban, N., Nissen, P., Hansen, J., Moore, P.B., and Steitz, T.A. (2000). Science, 289, 905-920.

[3] Gesteland, R.F., Cech T., and Atkins, J.F. (eds.). The RNA World: The Nature of Modern RNA Suggests a Prebiotic RNA World. $3^{\text {rd }}$ edn. (Cold Spring Harbor Laboratory Press, 2006).

[4] Read, R.J., et al. (2011). Structure, 19, 1395-1412.

[5] Davis, Ian W., Murray, Laura Weston, Richardson, Jane S., and Richardson, David C. (2004). Nucleic Acids Research, 32, W615-W619. 